\title{
Correspondence
}

\section{Neutralizing gp130 interferes with endothelial-mediated effects on glioblastoma stem-like cells}

\author{
Cell Death and Differentiation (2017) 24, 384; doi:10.1038/cdd.2016.163; published online 6 January 2017
}

Dear Editor,

Glioblastoma (GBM) is the most common and lethal primary brain tumor in adults. The aggressiveness of the disease partly relies on a subpopulation of tumor cells, termed as glioblastoma stem-like cells (GSCs) with a phenotype similar to that of normal neural stem cells such as multipotency and the ability to self-renewal. ${ }^{1,2}$ GSCs have been implicated in tumor initiation and growth, resistance to therapies, and recurrence. ${ }^{1-3}$ Additionally, it has been reported that GSCs reside in vascular niches in close contact with brain endothelial cells. These niches may regulate GSC selfrenewal, determine cell fate, and protect these cells from chemo- and radiation therapies. ${ }^{3,4}$ Accordingly, the localization of GSCs in close proximity to endothelial cells facilitates reciprocal communication, allowing notably the vascular niche to provide paracrine factors essential to maintain GSCs. ${ }^{4,5}$

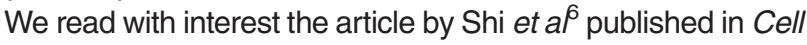
Death and Differentiation online on 14 October 2016. In this article, the authors implicate the glycoprotein gp130 and the tetraspanin $\mathrm{CD} 9$ as vital to maintaining the stem-like characteristics of GSCs. ${ }^{6}$ Employing RNA interference techniques, they observed a reduction in the stem-like properties of GSCs in the absence of gp130 when cultured in complete media. ${ }^{6}$ Our laboratory has also explored the role of gp130 in GSCs, using neutralizing antibodies (B-K5 clone) to pharmacologically alter its functions. To better reflect the in vivo endothelial microenvironment, our study was performed in human brain endothelial cell-conditioned serum-free mitogen-free media (EC-CM). ${ }^{5} \mathrm{We}$ too observed a drastic reduction in the stem-like properties in GSCs treated with the anti-gp130 blocking antibodies, assessed by both tumorsphere formation (Supplementary Figure S1a) and limiting dilution assays (Supplementary Figure S1b), as previously described. ${ }^{7,8}$ However, and in contrast to the Shi et al work, no significant impact of anti-gp130 blocking antibodies was observed when GSCs were grown in complete media (Supplementary Figure S1a-b). Moreover, blocking gp130 had no overt impact on cell viability in any of the four GSCs tested (data for GSC4 and GSC9 not shown) in either EC-CM (Supplementary Figure S1c) or complete media (Supplementary Figure S1c). ${ }^{9}$ Although our findings confirm the involvement of gp130 in stem maintenance, our data also suggest that the gp130 function in GSCs might vary along with cytokine and growth factor availability in the milieu.

The main differences in the two studies reside in the means employed in order to interfere with gp130 function: silencing versus blocking antibodies. Indeed, while Shi et af reported decreased stem characteristics and cell viability with gp130 silencing in complete medium, our study using antibodydirected targeting of gp130 did not recapitulate these findings. From these results, it is tempting to speculate that gp130 scaffolds a ligand-independent biased intracellular signaling in complete medium that could be affected by gp 130 silencing but not by antibodies. Conversely, the gp130 extracellular domainignited signaling action may be unmasked in EC-CM by neutralizing antibodies, while growth factor overload in complete medium might circumvent the need for gp130 extracellular domain-based signaling. Consequently, gp130 silencing or neutralization could target different signaling functions.

Taken together, our data reiterate the importance of gp130 in GSC maintenance, although therapeutic targeting of the gp130 complex alone might not lead to a full annihilation of its signaling functions as obtained through a genetic approach. Therefore, this indicates that we should remain cautious in our interpretations of such results as they may differ greatly when coming to the pre-clinical stage.

\section{Conflict of Interest}

The authors declare no conflict of interest.

Acknowledgements. The authors wish to thank Nicolas Bidère (SOAP team, Nantes, France) for careful reading of the manuscript. This research was funded by a grant from Région Pays-de-la-Loire and Nantes Métropole (Connect Talent), Ligue Nationale contre le Cancer (comité Loire-Atlantique, Maine-et-Loire, Morbihan, Sarthe, Vendée), Institut National du Cancer (INCA), Fondation ARC pour la Recherche contre le Cancer.

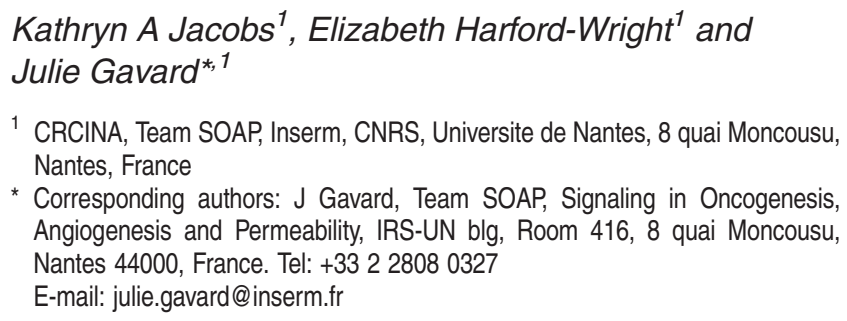

${ }^{1}$ CRCINA, Team SOAP, Inserm, CNRS, Universite de Nantes, 8 quai Moncousu, Nantes, France

* Corresponding authors: J Gavard, Team SOAP, Signaling in Oncogenesis, Angiogenesis and Permeability, IRS-UN blg, Room 416, 8 quai Moncousu, Nantes 44000, France. Tel: +3322808 0327

E-mail: julie.gavard@inserm.fr

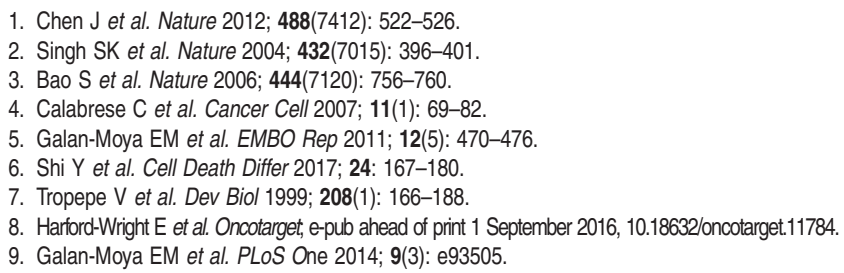

